33 STANDARD ASSESSMENT OF CHRONIC HEART FAILURE PATIENTS MAY UNDERESTIMATE SUBCLINICAL VOLUME OVERLOAD

M Shahzad, K McDonald, R Fox, E Shorten, A Brennan, C Halley, M Barrett, M Ledwidge. St. Vincent's University Hospital, Dublin, Ireland

\subsection{6/heartjn|-2021-ICS.33}

Introduction Volume control is critical in HF management. Clinical methods to assess volume status are neither sensitive nor specific enough to reliably predict volume overload. This lack of precision in volume assessment may leave patients at risk for decompensation or renal dysfunction. Ultrasound evaluation of inferior vena cava size has been useful to estimate and grade hemodynamic congestion in HF patients. Purpose We set out to examine the additional information provided by analysis of the IVC regarding volume status in a cohort of patients with chronic stable heart failure.We assessed NYHA class, clinical volume status, and inferior vena cava metrics.

Methods This is an ongoing observational study. This abstract presents data on the first 91. All patients had established HF and were attending for regular annual clinic review. Patients had a history obtained as a marker of clinical stability and clinical examination to assess for any signs of clinical congestion. These patients also had a complete Doppler-echocardiographic examination including assessment of E-e prime (E/e') and inferior vena cava including long axis diameter and collapsibility index ( IVCCI) as assessed by the reduction in size from baseline with inspiratory manoeuvre. Volume overload was defined as IVC diameter ( IVCD) $>2 \mathrm{~cm}$ with IVCCI $<$ $50 \%$. All patients had a natriuretic peptide assessment ( NT proBNP) and renal function measured.

Results To date sixty percent of the patients had reduced ejection fraction heart failure (LVEF $<50 \%$ ). Ninety five percent (87) had normal volume status on exam. The remaining four patients with volume overload on physical exam were omitted from further analysis. Thirty one patients (35\%) had Dopplerechocardiographic evidence of volume overload without signs of congestion on physical exam ( IVCD $\mathrm{M}=2.19, \mathrm{SD}=$ 0.49 and IVCCI $<50 \%)$. These patients had higher values of NT-proBNP $(\mathrm{M}=3,692, \mathrm{SD}=5,247 \mathrm{pg} / \mathrm{ml}, \mathrm{p}=0.005)$ and $\mathrm{E} / \mathrm{e}^{\prime} \mathrm{M}=11.5, \mathrm{SD}=3.3, \mathrm{P}=0.18$ ) compared with those with normal IVC metrics. Sixty five percent of the total group had atrial fibrillation. Within this subcohort those with IVC metrics of volume overload also had a higher value for NTproBNP ( $\mathrm{M}=4,266.7, \mathrm{SD}=5,904.5, \mathrm{p}<0.001)$ compared to those in AF a normal IVC metrics. Similar results were obtained in HF-pEF and HF-rEF cohorts.

Conclusion In conclusion, standard assessment of patients with stable chronic heart failure by symptom assessment and physical exam underestimates subclinical volume overload. This potentially leaves patients at higher risk for decompensation. Consideration of focused analysis of IVC and E-e prime in such patients in particular those with markedly elevated NP may be of value in diagnosing and managing this cohort.

\section{ASSESSMENT OF SPEED OF HEART RATE RECOVERY AS A MODIFIABLE RISK FACTOR THROUGH CARDIAC REHABILITATION}

R Armstrong, P Wheen, L Brandon, RA Kenny, A Maree. St James's Hospital, Dublin, Ireland
Introduction The rate of recovery of heart rate between 10 and 20 seconds following orthostatic challenge (HRR10-20) has been shown to be a risk factor for all-cause mortality. Cardiac rehabilitation has been shown to improve prognosis. We aim to determine if by assessing the physiological parameter of HRR10-20 in patients before, during and after cardiac rehabilitation, we can determine which patients are at greater risk of future events, and if this known risk factor for allcause mortality can be further classified as a modifiable risk factor in cardiac disease.

Methods A case-control study was performed from July 2019 until March 2020. Cases were patients aged $>18$ years of age who had PCI, CABG, TAVI or SAVR at the beginning of phase 2 of cardiac rehabilitation. A control group was taken from the same cohort of patients, but of whom turned down further cardiac rehabilitation. Assessment of HRR10-20 was performed at the beginning of rehabilitation, at a 6-weeks, and again at a 12-weeks. During active stand, real time heart rate, blood pressure and ECG recordings were taken via noninvasive digital photoplethysmography (Finometer, Finapres Medical systems, Arnhem, The Netherlands). Statistical analysis was performed using GraphPad Prism 9.0.2. Pearson's correlation coefficient was used to determine the relationship between change in HRR10-20 from baseline to 12 weeks and completion of the rehabilitation programme, and a Student's $\mathrm{T}$ test used to determine statistical significance of the difference between the two independent groups ( $p \leq 0.05$ was considered statistically significant).

Results Overall, 37 participants were recruited, 25 of which rehabilitation was commenced and completed versus 12 not commenced. Completion of cardiac rehabilitation was correlated with improvement of HRR10-20 from baseline to 12 weeks $(r=0.6104 \mathrm{p} \leq 0.0001)$. Through the 6 week and 12 week time periods, differences in HRR10-20 are noted between the two groups - at the 6-week time point the group in rehabilitation improved their HRR10-20 to $-5.74 \pm 1.91$, while the control group disimproved to $1.85 \pm 0.77$ $(\mathrm{p} \leq 0.0001)$. This pattern is repeated at the 12 -week interval, with the rehabilitation group maintaining a marginal improvement $-6.33 \pm 2.32$, and the control group deteriorating further to $4.05 \pm 1.27(p \leq 0.0001)$. HRR10-20 in the control group deteriorated between week 0 and week 6 by $5.94(p \leq 0.0001)$, between week 6 and 3 months this further disimproved by $2.2(\mathrm{p}=0.004)$. This is in comparison to a trend of improvement in HRR10-20 in the rehabilitation group; between week 0 and 6 weeks HRR10-20 improved by $-2.21 \quad(\mathrm{p}=0.1799)$,

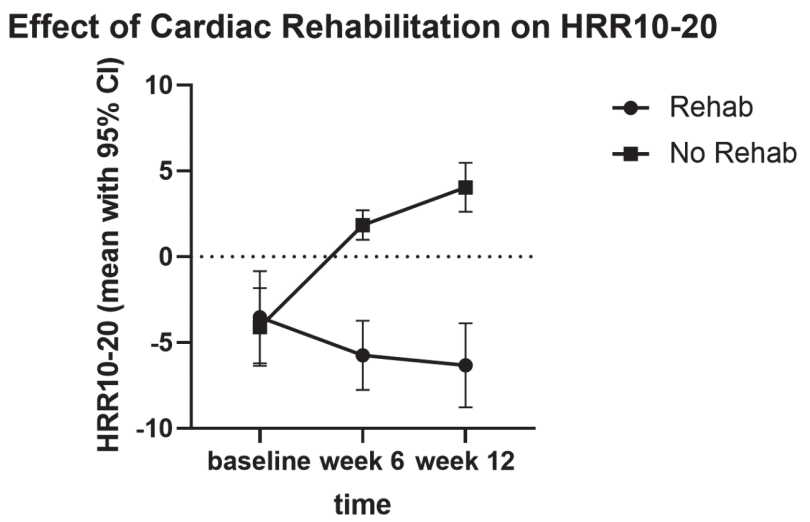

Abstract 34 Figure 1 
and between week 6 and 3 months HRR10-20 again improved by $0.6(\mathrm{p}=0.58)$ [figure 1 ].

Conclusions This study shows significant correlation between completing cardiac rehabilitation and improved autonomic function as measured with HRR10-20. This augmentation of autonomic function following cardiac rehabilitation may demonstrate how cardiac rehabilitation improves prognosis, and the use of autonomic function parameters as potentially modifiable risk factors in high-risk secondary prevention patients. The main limitation is the limited sample size; the trend observed of improved autonomic function through rehabilitation was in comparison to a group who, through not completing rehabilitation, had a deterioration in autonomic function. The trend of actual improvement in autonomic function may be significant in a larger cohort.

\section{General posters}

\section{INTRAVASCULAR LITHOTRIPSY-ASSISTED PCI FOR SEVERE CALCIFIC CORONARY DISEASE: EVALUATING THE IMPACT ON QUALITY OF LIFE AND OUTCOMES}

A Buckley, J McCormick, J Carey, R Armstrong, A Maree, M Hensey, S O'Connor, R Murphy, C Daly, J Cosgrave, I Pearson. St James's Hospital, Dublin, Ireland

\subsection{6/heartjnl-2021-ICS.35}

Introduction Severe coronary artery calcification (CAC) in patients undergoing percutaneous coronary intervention (PCI) is a common cause of procedural complications, lack of success, and stent failure. In this study we examine the utility of a novel technique to deal with this problem: intravascular lithotripsy (IVL) using the 'Shockwave' device.

\begin{tabular}{ll}
$\begin{array}{l}\text { Abstract } 35 \text { Table } 1 \\
\text { with coronary 'shockwave' intravascular lithotripsy }\end{array}$ & N $(\%)$ \\
\hline Baseline Demographics & $69 \pm 9$ \\
\hline Age & $39(83)$ \\
Male & $19(40)$ \\
Diabetes Mellitus & $15(32)$ \\
NIDDM & $4(8)$ \\
IDDM & \\
Renal Impairment & $18(38)$ \\
None & $17(36)$ \\
Moderate & $6(13)$ \\
Severe & $6(13)$ \\
Dialysis dependent & $34(72)$ \\
PCI & $4(8)$ \\
CABG & $3(6)$ \\
CVA/TIA & $36(77)$ \\
Smoking history & \\
Clinical presentation & $28(60)$ \\
Stable angina & $2(4)$ \\
Chronic Total Occlusion & $8(17)$ \\
Unstable angina & $9(19)$ \\
NSTEMI & $2(4)$ \\
STEMI & $2.49(0.5-13)$ \\
EuroSCORE II, median (range) & $23(4-61)$ \\
SYNTAX, median (range) &
\end{tabular}

Methods A retrospective single centre study was conducted of consecutive patients treated with coronary IVL between January and October 2020. Baseline demographics were obtained from electronic patient records and SYNTAX scores were calculated from index coronary angiograms. Procedural success was verified by independent review of the angiogram and defined as (1) adequate stent expansion, (2) no significant residual stenosis and (3) TIMI III flow in all major branches. Target lesion revascularisation (TLR) was defined as any unplanned repeat PCI of the target lesion or bypass of the target vessel performed for in-stent restenosis (ISR) or other complication of the target lesion. Clinical outcomes including Canadian Cardiovascular Society (CCS) angina classification were assessed at virtual clinical follow-up.

Results 47 patients were treated with IVL during the study period. Baseline demographics and procedural characteristics are seen in tables 1 and 2, respectively. All patients had circumferential or near-circumferential calcium as determined by coronary angiography \pm intracoronary imaging. $57 \%$ had treatment of a de novo lesion, $32 \%$ had PCI to a previously

Abstract 35 Table 2 Procedural characteristics of patients treated with coronary 'shockwave' intravascular lithotripsy

\begin{tabular}{|c|c|}
\hline Procedural characteristics & N (\%) \\
\hline Mean IVL balloon diameter (mm) & 3.5 \\
\hline Stent deployed & $34(72)$ \\
\hline Mean stent length, where deployed (mm) & 51 \\
\hline \multicolumn{2}{|l|}{ Target vessel } \\
\hline RCA & $17(36)$ \\
\hline LMS & $2(4)$ \\
\hline LAD & $9(19)$ \\
\hline $\mathrm{LCX}$ & $4(8)$ \\
\hline Multi-vessel & $15(32)$ \\
\hline \multicolumn{2}{|l|}{ Access } \\
\hline Radial & $31(66)$ \\
\hline Femoral & $14(30)$ \\
\hline Both & $2(4)$ \\
\hline \multicolumn{2}{|l|}{ Adjunctive therapies } \\
\hline Rotablation & $8(17)$ \\
\hline Laser atherectomy & $3(6)$ \\
\hline Diathermy & $1(2)$ \\
\hline \multicolumn{2}{|l|}{ Intracoronary imaging } \\
\hline IVUS & $39(83)$ \\
\hline OCT & $4(8)$ \\
\hline Procedural success & $44(94)$ \\
\hline Significant residual stenosis in de novo lesion & $1(2)$ \\
\hline Resistant stent under-expansion & $1(2)$ \\
\hline Dissection with no-reflow & $1(2)$ \\
\hline \multicolumn{2}{|l|}{ Complications } \\
\hline Coronary dissection & $6(13)$ \\
\hline Stented & $2(4)$ \\
\hline Conservative & $4(8)$ \\
\hline Coronary perforation & $1(2)$ \\
\hline \multicolumn{2}{|l|}{ Target Lesion Revascularization (TLR) } \\
\hline CABG & $2(4)$ \\
\hline Planned & $1(2)$ \\
\hline Unplanned & $1(2)$ \\
\hline Planned/staged-PCI & $11(23)$ \\
\hline Same vessel as IVL & $10(21)$ \\
\hline Alternate vessel & $1(2)$ \\
\hline
\end{tabular}

